\title{
Substrate Effect on the Melting Temperature of Thin Polyethylene Films
}

\author{
Y. Wang, ${ }^{1}$ M. Rafailovich, ${ }^{1, *}$ J. Sokolov, ${ }^{1}$ D. Gersappe, ${ }^{1}$ T. Araki, ${ }^{2}$ Y. Zou, ${ }^{2}$ A. D. L. Kilcoyne, ${ }^{3}$ H. Ade,${ }^{2}$ \\ G. Marom, ${ }^{4}$ and A. Lustiger ${ }^{5}$ \\ ${ }^{1}$ Department of Materials Science and Engineering, SUNY at Stony Brook, Stony Brook, New York 11794-2275, USA \\ ${ }^{2}$ Department of Physics, NCSU, Raleigh, North Carolina 27695, USA \\ ${ }^{3}$ Advanced Light Source, LBNL, Berkeley, California 94720, USA \\ ${ }^{4}$ Department of Applied Chemistry, The Hebrew University of Jerusalem, Jerusalem 91904, Israel \\ ${ }^{5}$ ExxonMobil Research and Engineering Company, Annandale, New Jersey 08801, USA
}

(Received 26 April 2005; published 17 January 2006)

\begin{abstract}
Strong dependence of the crystal orientation, morphology, and melting temperature $\left(T_{m}\right)$ on the substrate is observed in the semicrystalline polyethylene thin films. The $T_{m}$ decreases with the film thickness decrease when the film is thinner than a certain critical thickness, and the magnitude of the depression increases with increasing surface interaction. We attribute the large $T_{m}$ depression to the decrease in the overall free energy on melting, which is caused by the substrate attraction force to the chains that competes against the interchain force which drives the chains to crystallization.
\end{abstract}

DOI: 10.1103/PhysRevLett.96.028303

PACS numbers: 82.35.Gh, 64.70.Dv

The crystallization of polymer thin films on a solid substrate has been studied for decades due to its importance in determining interfacial properties of coatings, such as adhesion, wear resistance, and lubrication [1,2]. Numerous groups have demonstrated that the rate of crystallization, crystal orientation, and density of nucleation points can be very different for the thin films from the bulk [1-5]. We previously observed that the degree of crystallinity of polyethylene (PE) remained high even in films as thin as $15 \mathrm{~nm}$ [5]. A novel technique, lateral scanning force modulation, was used to determine the melting point of the semicrystalline thin films. We found that these films melt $5-38^{\circ} \mathrm{C}$ below the bulk values, depending on film thickness. This large depression cannot be explained classically using the Thomson-Gibbs equation [5]. Although it is well known that $T_{m}$ of inorganic crystals is depressed near a free surface [6,7], in the semicrystalline polymers it appears as if this effect persists close to the substrate. Furthermore, this large decrease was also found to be counterintuitive when compared to the results for small molecule systems, whose $T_{m}$ was increased near an attractive surface [8]. Hence, we propose that, in analogy to the previously reported interface effects on the glass transition $\left(T_{g}\right)$ of polymer films [9], these interactions could also be implicated in changing $T_{m}$. Unlike glass transition, melting is a well-defined first order transition and is an intrinsic component defining the material. Hence it is important to obtain a fundamental understanding at the molecular level of the factors which differentiate the amorphous to crystalline transition in complex molecular fluids vs simple liquids.

We performed a comprehensive study where we focused on the effects of surface interactions, as well as crystalline orientation and morphology, on the melting point of crystalline films as a function of thickness. We investigated the chain orientation using near edge x-ray absorption fine structure (NEXAFS) spectroscopy, measured the interfacial tension using the Owens-Wendt-Kaelble (OWK) approach [10], and compared $T_{m}$ for polymers with different branching ratios. We then showed that the $T_{m}$ depression could be correlated with the change of the free energy on melting of the polymer chains resulting from adsorption to attractive interfaces above $T_{m}$.

The polymers used were linear low and medium density polyethylene (ExxonMobil Corp.), designated as B-3 and B-5, respectively, with hexene as the comonomer. The two polymers have the same molecular weight $\left(M_{w}=50000\right.$, $\left.M_{w} / M_{n}=2\right)$ but different densities $\left(\rho_{\mathrm{B}-3}=0.92, \rho_{\mathrm{B}-5}=\right.$ $0.95 \mathrm{~g} / \mathrm{cm}^{3}$ ), branching ratios (20 branches for B-3 and 2.3 branches for B-5 per 1000 backbone atoms), and bulk melting points $\left(117^{\circ} \mathrm{C}\right.$ for B-3 and $132^{\circ} \mathrm{C}$ for B-5). Thin films were prepared by spin coating the polymer/toluene solutions onto different substrates at above $100^{\circ} \mathrm{C}$, followed by slow cooling from $140^{\circ} \mathrm{C}$ to room temperature in vacuum oven. Three substrates were used: polished silicon (111) wafers passivated in dilute HF solution; polished silicon wafers coated with aluminum films ( $400 \AA)$; and polyimide (PI) sheets. The surface energies of these substrates were determined by measuring the contact angle for two liquids (diiodomethane and water) and combining Young's equation $\left(\gamma_{s}=\gamma_{l} \cos \theta+\gamma_{s l}\right)$ and the OWK equation $\quad\left[\gamma_{l s}=\gamma_{l}+\gamma_{s}-2\left(\gamma_{s}^{d} \gamma_{l}^{d}\right)^{1 / 2}-2\left(\gamma_{s}^{p} \gamma_{l}^{p}\right)^{1 / 2}\right]$ [10]. The surface tension of the PE melt was estimated to be $30 \mathrm{~mJ} / \mathrm{m}^{2}$ [11]. The interfacial energies between the PE and the substrates were then calculated using the OWK equation; the values are listed in Table I. As expected, we can see that HF passivated silicon wafer has a very small polar component. However, the imide groups in the polyimide produce a significant polar component to the surface tension. As a result, the interfacial tension between the 
TABLE I. Interfacial energy between B-3 melt and different substrates. $\gamma_{s}^{d}$ and $\gamma_{s}^{p}$ stand for the dispersive part and the polar part of the surface energy, respectively.

\begin{tabular}{lccc}
\hline \hline & $\gamma_{s}^{d}\left(\mathrm{~mJ} / \mathrm{m}^{2}\right)$ & $\gamma_{s}^{p}\left(\mathrm{~mJ} / \mathrm{m}^{2}\right)$ & $\gamma_{\mathrm{PE} / \text { substrate }}\left(\mathrm{mJ} / \mathrm{m}^{2}\right)$ \\
\hline Silicon & 48.71 & 3.98 & 5.60 \\
Aluminum & 50.39 & 5.13 & 7.05 \\
Polyimide & 46.25 & 10.23 & 11.98 \\
PE & 30 & 0 & \\
\hline \hline
\end{tabular}

nonpolar PE and the hydrogen-passivated Si surface is the lowest, while the interfacial energy on the more polar PI is the highest.

Figure 1 shows the scanning probe microscopy (SPM) height and friction images for a thick and a thin B-3 film on each of the three substrates. We can see that the crystals in the films on the Si substrate are highly ordered, with clear spherulitic structure in the thicker film, but with axialitic structures in the thinner film. The friction images indicate that a transition from mainly edge-on to mainly flat-on lamellar orientation may be occurring with decreasing film thickness. On the $\mathrm{Al}$ and PI substrate the crystalline structure is not as well ordered. Crystallites are rather randomly distributed. Furthermore, little difference in ori-
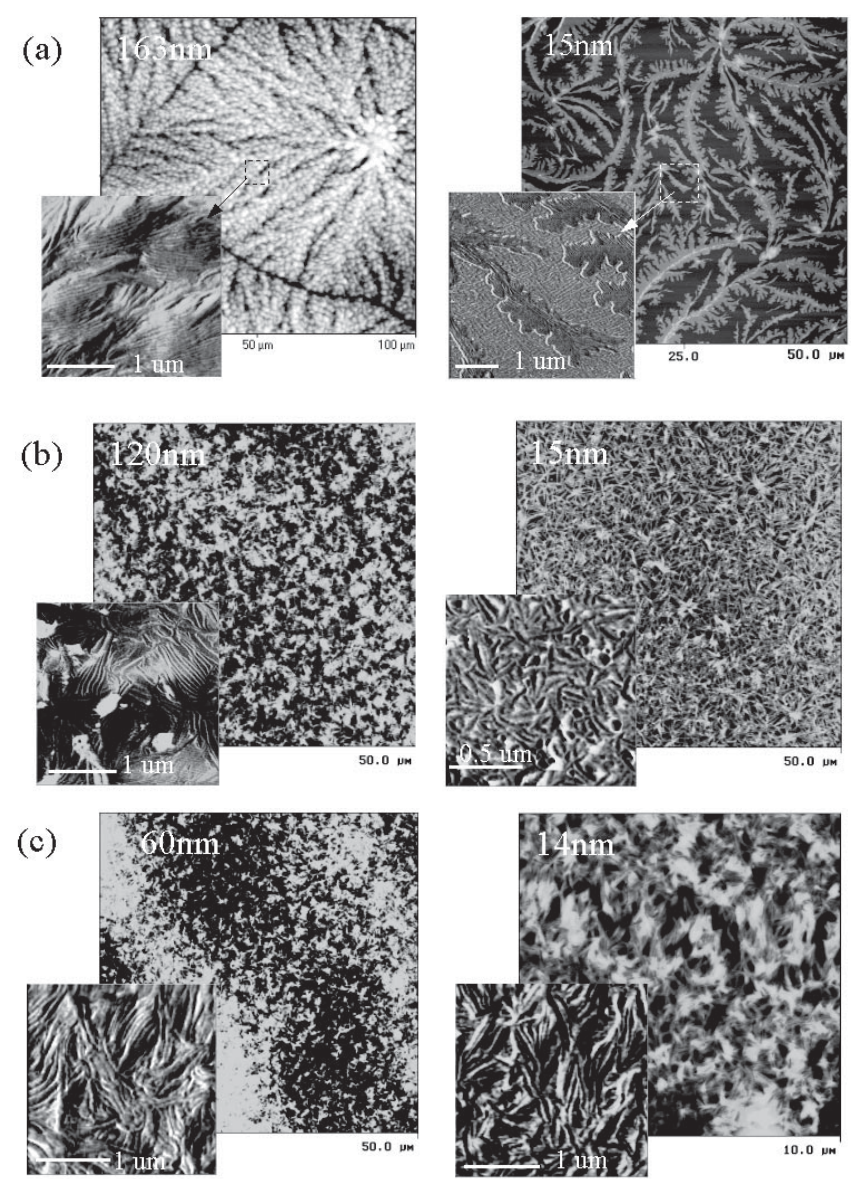

FIG. 1. SPM images of B-3 films on (a) silicon, (b) aluminum, and (c) polyimide substrates. The large images are height image; the small insets are friction images. entation is observed with decreasing film thickness, and in all cases edge-on lamellae are observed. Similar results are observed in the B-5 films, namely, that the morphology of the films on the silicon substrate changes significantly in the ultrathin films, while no change is seen on the $\mathrm{Al}$ and PI substrates. A more direct orientation measurement is obtained using scanning transmission x-ray microscopy (STXM). This technique collects NEXAFS spectra with the X-ray beam linearly polarized in the film plane from spots of $40 \mathrm{~nm}$ in diameter [12]. The STXM images and the corresponding NEXAFS spectra of a thick and a thin PE sample crystallized on $\mathrm{Si}$ wafer and transferred onto a TEM grid are shown in Fig. 2. In the thick sample, the two NEXAFS spectra taken at different spots within the same spherulite are quite different. Fibrils lying along the photon polarization alignment (spot 1) show strong $\mathrm{C}-\mathrm{H}$ features near $288 \mathrm{eV}$ and relatively weak C-C features near $293 \mathrm{eV}$ [13]. Since the C-H absorption is maximized for polarization perpendicular to the chain while the $\mathrm{C}-\mathrm{C}$ absorption is maximized for polarization along the chain [13], the result is consistent with edge-on lamella with C-C bonds parallel to the substrate plane and perpendicular to the fibril direction. In contrast, the $25 \mathrm{~nm}$ thick film shows only very strong $\mathrm{C}-\mathrm{H}$ features and suppressed $\mathrm{C}-\mathrm{C}$ features irrespective of location. This supports flat-on lamellae with the $\mathrm{C}-\mathrm{C}$ backbone normal to the substrate surface. The orientation of the crystals on the aluminum substrate is further confirmed with grazing incidence reflection Fourier transformed infrared spectroscopy, which shows crystal-

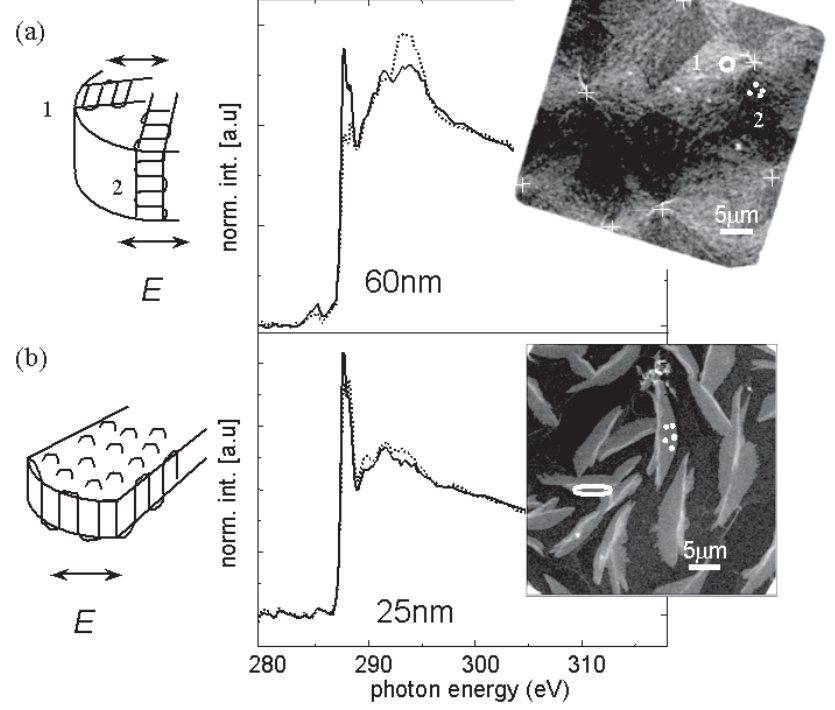

FIG. 2. Orientation measurement by NEXAFS of B-5 thin films with thickness of (a) $\sim 60 \mathrm{~nm}$ and (b) $\sim 25 \mathrm{~nm}$. NEXAFS spectra from locations as indicated in the optical density images. The centers of the spherulites in (a) are indicated by a white cross. The observed dichroism indicates predominantly edge-on lamellae for (a) and flat-on lamellae for (b). The scheme of the chain orientation vs electron vector is shown on the left. 
line peak but no clear orientation change [14]. From these results, we infer that the surface can act as a nucleating agent for a specific orientation when attractive interactions are present with the polymer.

The melting point was measured by shear modulus force microscopy (SMFM) [15]. In this technique we measure the lateral deflection amplitude $\Delta x$ of the SPM tip, which has the following relationship with the lateral modulus $G$ of the film: $\Delta x \propto G^{-2 / 3}[5,15]$. Since $G$ decreases by orders of magnitude when the film melts over a span of $1-2{ }^{\circ} \mathrm{C}$, the signal is strong and sharp. The amplitudetemperature curves of the thick and the thin B-3 films on the three substrates are shown in Fig. 3. For the thick films, the amplitude rises abruptly around $T=117^{\circ} \mathrm{C}$ or $T_{m}$ (bulk) of B-3. For the ultrathin films, the $T_{m}$ differed greatly for the films on three substrates, and in all cases was lower than the bulk value. The $T_{m}$ for the B-3 films vs film thickness on three substrates is summarized in Fig. 4(a). The magnitude of the depression was the largest on the Si substrate: $38^{\circ} \mathrm{C}$ and $22^{\circ} \mathrm{C}$ or $12^{\circ} \mathrm{C}$ on the $\mathrm{Al}$ or PI substrates, respectively. The critical thickness for the onset of the $T_{m}$ depression can be as large as $72 \mathrm{~nm}$ for the films on Si substrate. Comparing the surface interactions in Table I we find that the magnitude of the depression increases with increased surface interactions. In Fig. 3(b), $T_{m}$ for the B-5 films on Si and PI substrates is plotted as a function of film thickness. The same trend is apparent for this polymer, though the magnitude of the depression, $18^{\circ} \mathrm{C}$ on $\mathrm{Si}$ and $5^{\circ} \mathrm{C}$ on PI, is smaller than that for B-3.

Following the same derivation for Thomson-Gibbs equation by Young et al. [16], the expression for the melting point of the finite size crystal under confinement

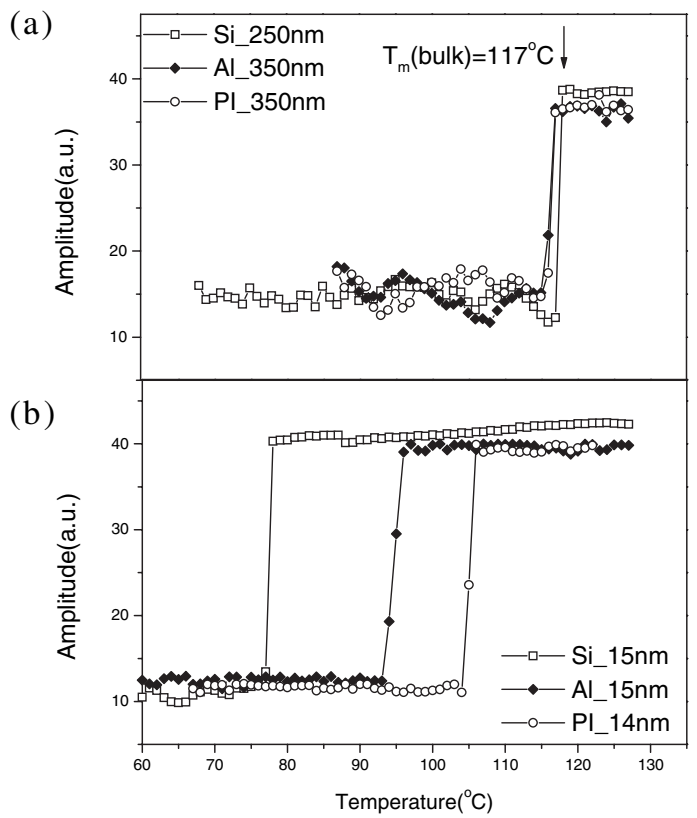

FIG. 3. SMFM curves for B-3 films on silicon, aluminum, and polyimide substrates (a) for films with thickness above $200 \mathrm{~nm}$ and (b) for thin films with thickness below $20 \mathrm{~nm}$. is [5]

$$
T_{m}=T_{m}^{0}\left(1-\frac{2 \gamma_{e}}{l \Delta H_{0}}-\frac{2 \gamma_{l}}{x \Delta H_{0}}\right),
$$

where $T_{m}^{0}$ is the melting point of the ideal infinitely large crystal expressed as $T_{m}^{0}=\frac{\Delta H_{0}}{\Delta S_{0}}$, since melting occurs when $\Delta g=\Delta H_{0}-T \Delta S_{0}=0$, where $\Delta g$ is the free energy change per unit volume, and $\Delta H_{0}$ and $\Delta S_{0}$ are the enthalpy and entropy of melting per unit volume, respectively; $x$ and $l$ are the lamellar width and thickness, respectively; $\gamma_{e}$ and $\gamma_{l}$ are the corresponding surface energy [Fig. 4(c)]. The thickness effect on the $T_{m}$ is accounted for through the confinement on the $l$ or $x$ dimension, depending on the lamellar orientation [Fig. 4(c)]. However, using the constant bulk value of $T_{m}^{0}$ to fit the data would lead to a more gradual $T_{m}$ depression with film thickness decrease [5]; thus we expect that $T_{m}^{0}$ also changes in the thin films due to the substrate interaction.

On the attractive substrate, the free energy change on melting per unit volume, $\Delta g_{f}$, will include substrate interactions $(\Delta F): \Delta g_{f}=\Delta H_{0}-T_{m} \Delta S_{0}+\Delta F$, and hence

$$
T_{m}^{0^{\prime}}=\frac{\Delta H_{0}+\Delta F}{\Delta S_{0}} .
$$

In semicrystalline polymers, the main force that drives

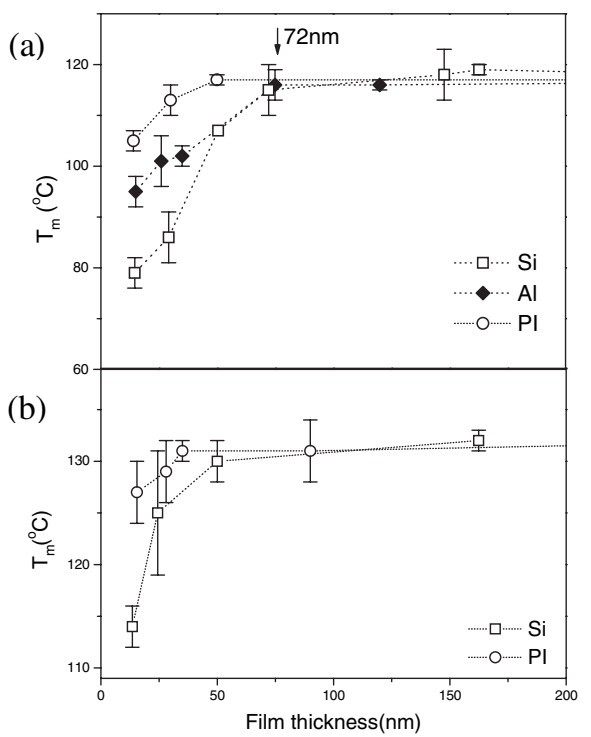

(c)

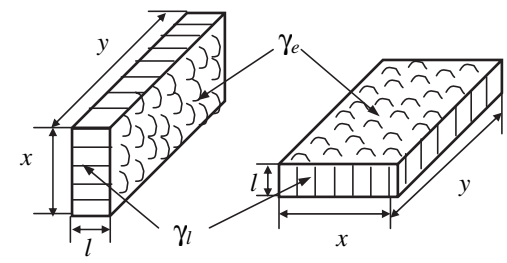

FIG. 4. (a) Melting point of B-3 vs film thickness on silicon, aluminum, and polyimide substrates. (b) Melting point of B-5 vs film thickness on silicon and polyimide substrates. (c) Confinement effect on the lamellar size for edge-on and flat-on lamellar orientation. 
chain ordering in the crystalline state is the attraction among the nonbonded monomers [17]. This decrease in internal energy is counterbalanced by the entropic terms which resist the order $[17,18]$. When an attractive interface is present, pinning of the chains adjacent to the surface occurs. This can then affect crystallization by changing both the entropic and enthalpic contributions. The entropic barrier the chains must overcome during crystallization is decreased since the chains adjacent to the interface are already slightly confined, even above $T_{m}$, relative to in their unperturbed Gaussian configurations. However, this reduction in melting entropy is not expected to be significant since the chains in the melt tend to be adsorbed onto the substrate randomly. On the other hand, because the surface is incapable of accommodating the crystal lattice of the polymer, chain folding and crystallization require desorbtion from the surface and rearrangement at $T \sim T_{m}$. Hence, when melting transition occurs on the attractive substrate, the melting enthalpy will be reduced relative to the bulk value due to the adsorption energy in the melt state [19]. When the enthalpy term dominates, the combining effect of the entropy and enthalpy leads to a decrease in the overall free energy on melting, and $\Delta F$ in Eq. (2) is negative, hence $T_{m}^{0^{\prime}}<T_{m}^{0}$. In another word, the substrate attraction force tends to break the order of the chains in the crystalline state by randomly adsorbing and pinning the chains, which will lead to the system to melt at a lower temperature. And this is unique in the long chain molecule system since the tendency for randomly adsorbing the chain and breaking the order is less possible for the small molecules due to the short chain length. The importance of these forces will depend on the film thickness and the volume fractions of chains that have contact with the surface, and this fraction will obviously diminish as the film thickness increases [20]. Finally, the perturbation induced by the attractive surface is known to extend for large distances into the films and was observed to affect the dynamics of chains which do not have direct contacts with the surface [21]. This is reasonable since melting is a highly collective process; the onset of the surface induced melting point depression will also occur, as is seen in Fig. 4, for distances large compared to $R_{g}$.

The effect of the branching ratio on the depression of $T_{m}$ is more subtle. Chains with higher branching ratios are somewhat stiffer due to the restriction of the rotation and bending of the backbone chains by the side chains [16]. According to the simulation works by Sintes et al., the stiffer chains are more easily adsorbed by the substrate and thus will have more contacts with the substrate [22]. Hence a higher branching ratio can be more effective in depressing the melting point.

In summary, we have shown that a decrease in melting point for semicrystalline polymers occurs on the interactive surfaces. The degree of orientation is increased with increasing surface interactions. Contrary to small molecule systems where an increase is observed, in long chain molecules the depression in the melting point increases with increasing surface interactions. The substrate provides a source of interactions with the individual polymer chains that compete with the forces that drive the system to crystallization. From the thermodynamic point of view, this interaction leads to a decrease in the overall free energy on melting. This has the effect of reducing the temperature at which the system melts.

We thank the NSF (DMR-0080604) program for support, and Dr. Andy Lovinger and Dr. Harry Frisch for invaluable advice and discussions. We thank Dr. Shouren Ge for great help with the SMFM measurement. STXM was performed at BL5.3.2. of the Advanced Light Source. Work at NCSU was partially supported by DOE Grant No. DE-FG02-98ER45737.

*Corresponding author.

Electronic address: mrafailovich@notes.cc.sunysb.edu

[1] C. W. Frank et al., Science 273, 912 (1996).

[2] K. Cho, D. Kim, and S. Yoon, Macromolecules 36, 7652 (2003).

[3] H. Schönherr and C. W. Frank, Macromolecules 36, 1199 (2003).

[4] G. Reiter and J. U. Sommer, J. Chem. Phys. 112, 4376 (2000).

[5] Y. Wang et al., Macromolecules 37, 3319 (2004).

[6] L. H. Liang, M. Zhao, and Q. Jiang, J. Mater. Sci. Lett. 21, 1843 (2002).

[7] J.F. van der Veen, Surf. Sci. 433, 1 (1999).

[8] J. Cousty and A. Marchenko, Surf. Sci. 520, 128 (2002).

[9] J. A. Forrest, K. Dalnoki-Veress, and J. R. Dutcher, Phys. Rev. E 56, 5705 (1997).

[10] D. Y. Kwok and A. W. Neumann, Adv. Colloid Interface Sci. 81, 167 (1999).

[11] S. Wu, J. Macromol. Sci, Part C 10, 1 (1974).

[12] A. L. D. Kilcoyne et al., J. Synchrotron Radiat. 10, 125 (2003).

[13] T. Ohta et al., Phys. Scr. 41, 150 (1990); A. Schöll et al., Chem. Phys. Lett. 370, 834 (2003).

[14] H. Hagemann, Macromolecules 22, 3600 (1989); M. Tasumi and T. Shimanouchi, J. Chem. Phys. 43, 1245 (1965).

[15] S. Ge et al., Phys. Rev. Lett. 85, 2340 (2000); Y. Zhang et al., Polymer 44, 3327 (2003).

[16] R. J. Young and P. A. Lovell, Introduction to Polymers (Chapman \& Hall, London, 1991), 2nd ed., Chap. 4.

[17] P. Welch and M. Muthukumar, Phys. Rev. Lett. 87, 218302 (2001).

[18] M. Muthukumar, Eur. Phys. J. E 3, 199 (2000).

[19] A.P. Angelopoulos, J. Colloid Interface Sci. 243, 292 (2001).

[20] Y. Pu et al., Phys. Rev. Lett. 87, 206101 (2001).

[21] X. Zheng et al., Phys. Rev. Lett. 79, 241 (1997); B. Frank et al., Macromolecules 29, 6531 (1996).

[22] T. Sintes, K. Sumithra, and E. Straube, Macromolecules 34, 1352 (2001). 